\title{
La cellule souche hématopoïétique humaine : du mythe à la réalité
}

"Cellule mère du sang " et réalisme financier

La cellule souche hématopoiétique humaine est restée longtemps un mythe ; elle est en passe de devenir une réalité. Lorsque la société de biotechnologie Systemix, dont le travail dans le domaine de la cellule souche est remarquable [1], déposa un brevet sur... la cellule souche, les journaux américains qui aiment les grands mythes annoncèrent en gros titres la découverte de la "cellule mère du sang ": les actions de la société montèrent brusquement. Ce brevet scandalisa bon nombre de chercheurs qui, depuis plus de trente ans, avaient développé les outils ayant permis la caractérisation et la purification de cette cellule. La réalité, c'est que les cellules souches ne sont probablement pas constituées par une population homogène, mais qu'elles regroupent différentes souspopulations ayant des potentiels de prolifération et de différenciation variables pour produire les progéniteurs lympho-myéloïdes.

Une cellule au repos pour préserver un énorme potentiel de prolifération et de différenciation

Les cellules souches sont rares, environ 1 pour 10000 parmi les cellules mononucléées de la moelle osseuse et, bien qu'étant à l'origine du système cellulaire le plus prolifératif, elles restent la plupart du temps sans se diviser. Cet état quiescent protège le patrimoine génomique d'accidents possibles au cours de la division cellulaire. Rareté et quiescence ont été deux causes importantes de la difficulté de l'étude de ces cellules.

En observant l'édifice remarquablement équilibré auquel les cellules souches donnent naissance, on pourrait penser que celles-ci sont programmées génétiquement dans les voies de la différenciation hématologique. La réalité ne semble pas si simple. En effet, l'orientation de la cellule souche vers une lignée déterminée plutôt qu'une autre semble s'opérer de façon aléatoire, dite stochastique, selon la théorie de J.E. Till et E.A. McCulloch [2], théorie bien étayée par les expériences de M. Ogawa [3]. Cette théorie peut choquer les esprits rationalistes qui admettent mal qu'un système réglé de façon aussi précise puisse obéir aux lois du hasard. Mais si le hasard est à l'origine de certains choix dans la différenciation, la mise en évidence de régulations internes, en aval des progéniteurs, explique l'homéostasie. Dans notre laboratoire, B. Panterne a récemment montré un rétrocontrôle des cellules mûres monocytaires sur leurs progéniteurs [4]. Ce rétrocontrôle se fait par l'intermédiaire d'un facteur de croissance, le macrophage-colony-stimulating factor ou CSF-1 sécrété par les monocytes mûrs, qui contrôle négativement son récepteur sur les progéniteurs myélo-monocytaires. Ce rétrocontrôle diminue la production de monocytes au profit des granulocytes. L'équipe de A.W. Burgess avait suggéré une hiérarchie des différents colony stimulating factors (CSF) et interleukines dans le rétrocontrôle de l'expression des récepteurs de progéniteurs [5]. Ce contrôle peut s'exercer sur le récepteur propre de la cytokine (down-regulation) ou sur le récepteur d'une autre cytokine (downmodulation). On voit donc que, si la théorie stochastique postule un mécanisme aléatoire de la différenciation dans le compartiment de la cellule souche, il existe cependant des mécanismes de régulation jouant sur les progéniteurs dérivés de cette cellule. Il convient de ne pas avoir une lecture dogmatique des différentes théories expliquant la différenciation de la cellule souche. L'interprétation de certaines expériences peut être modifiée ultérieurement à la lumière de données nouvelles. C'est ainsi que les résultats de certaines expériences de D. Metcalf, expliquées par l'auteur en termes déterministes [6], peuvent être actuellement réinterprétés par des mécanismes de rétrocontrôle semblables à ceux que nous venons de décrire [7].

Un problème intéressant de biologie cellulaire posé par la cellule souche est celui de son autorenouvellement. L'autorenouvellement est un dogme qui n'est soutenu encore par aucune expérience satisfaisante. La cellule souche peut-elle s'autorenouveler sans que l'une au moins des deux cellules filles ne se différencie? Les meilleures expériences d'autorenouvellement montrent qu'il resterait limité à quelques divisions dans les systèmes de culture actuellement disponibles. Mais cela ne veut pas dire que nous n'ayons pas à découvrir des mécanismes plus physiologiques régissant l'autorenouvellement ou amplification sans différenciation, ce qui permettrait de produire pour chaque individu une réserve de cellules souches utilisable en cas de besoin.

Des inhibiteurs, aussi importants que les facteurs de croissance, pour contrôler la cellule souche

La recherche de nouvelles cytokines a également alimenté le mythe de la cellule souche. Le terme de stem cell factor donné au steel factor, kit ligand ou mast cell factor correspond plus à des besoins publicitaires qu'à une réalité scientifique. En effet, le steel factor n'a pas une action spécifique sur la cellule souche, il est incapable de la sortir de l'état de quiescence, il est inefficace par lui-même. En revanche, il est un très remarquable costimulateur agissant en coopération ou en synergie avec de nombreuses autres cytokines pour activer différents types de progéniteurs proches de la cellule souche [8].

Devant l'inefficacité des cytokines à sortir la cellule souche de la phase de 
quiescence Go, notre laboratoire a cherché à voir si celle-ci n'était pas maintenue dans cet état par des inhibiteurs extracellulaires, ou des facteurs de contrôle négatif intracellulaires. On connaissait déjà des inhibiteurs comme le TGF- $\beta$ (transforming growth factor $\beta$ ), le MIP-1 $\alpha$ (macrophage inflammatory protein-1 $\alpha$ ) et d'autres facteurs qui ont fait l'objet de la très intéressante " $3^{\mathrm{e}}$ conférence internationale sur la régulation négative de la prolifération et de la différenciation hématopoïétique " qui s'est tenue à Paris en avril 1993 [9].

On connaissait également les gènes suppresseurs de tumeur dont l'expression et la fonction dans le compartiment de la cellule souche restaient à démontrer. Étant une cellule rare, la cellule souche se prête mal à certaines techniques de biologie moléculaire qui nécessitent un grand nombre de cellules. Il faut recourir à l'hybridation in situ ou à la PCR inverse pour étudier l'expression des gènes qui la contrôlent. C'est aussi en développant des oligonucléotides antisens pour des inhibiteurs et pour des gènes suppresseurs de tumeur que nous avons réussi à étudier la fonction de gènes qui maintiennent le compartiment de la cellule souche à l'état quiescent. Cette stratégie nous a permis de contrôler la sortie de la phase Go de cellules CD34 + CD38 - appartenant au compartiment de la cellule souche et nous avons ainsi réussi à les faire se diviser in vitro [10]. En effet, en bloquant l'expression d'inhibiteurs comme le TGF- $\beta 1$ ou le produit de gènes suppresseurs de tumeurs tels que le gène $\mathrm{R} b$ de susceptibilité au rétinoblastome, nous avons pu activer des progéniteurs plus précoces que ceux que nous pouvions cultiver auparavant avec les meilleures combinaisons de cytokines. A l'aide de cultures de cellules isolées provenant du compartiment de la cellule souche, nous avons pu montrer que des oligonucléotides antisens du TGF- $\beta 1$ bloquaient une production autocrine de cet inhibiteur. La cellule souche posséderait donc la machinerie nécessaire à son maintien à l'état quiescent. Grâce à cette stratégie des oligonucléotides antisens pour des inhibiteurs ou des gènes sup$\mathrm{m} / \mathrm{s} n^{\circ} 10$ vol. 9 , octobre 93 presseurs, nous pouvons non seulement étudier les contrôles de la phase Go, mais évaluer le potentiel hématopoïétique des cellules que l'on souhaiterait utiliser à des fins cliniques. Conséquence de la mise en cycle des cellules, nous avons également pu doubler l'efficacité du transfert du gène nls lac $\mathrm{Z}$ dans des progéniteurs à l'aide d'une construction rétrovirale.

La purification de la cellule souche peut se faire par des sélections positives de cellules portant certains antigènes (CD34, Thy-1) combinées à des sélections négatives pour des antigènes de différenciation (CD38, etc.). L. Terstappen a montré que le compartiment de la cellule souche était inclus dans la souspopulation CD34+ CD38- et que la population des cellules CD34+ CD38- HLA DR - de la moelle osseuse fotale contenait une cellule souche pouvant se différencier non seulement vers les lignées lymphomyéloïdes mais, chose surprenante, également vers des cellules stromales [11]. Nous avons montré que ce sont les cellules CD34+CD38- et non les cellules CD34+CD38+ qui sont activées par l'oligonucléotide antisens TGF- $\beta 1$ [12], et donc que le compartiment de la cellule souche est capable de produire cet inhibiteur de la prolifération, à l'instar d'autres cellules, telles les cellules stromales.

Coupez le cordon ombilical... et conservez le sang qu'il contient : vous permettrez la greffe d'un adulte

Depuis la première greffe réussie par E. Gluckman et H. Broxmeyer à l'hôpital Saint-Louis, le sang de cordon ombilical est de plus en plus utilisé pour greffer des enfants. La question se pose de savoir quelle quantité de sang de cordon ombilical est suffisante pour greffer un adulte. Pour répondre à cette question, nous avons comparé le potentiel hématopoiétique d'un sang de cordon ombilical de taille moyenne avec celui d'un prélèvement de moelle osseuse de taille suffisante pour greffer un adulte. Nous avons purifié les cellules CD34 + CD38du sang de cordon et de la moelle osseuse; nous les avons fait proliférer en culture liquide à long terme en bloquant la production autocrine de TGF- $\beta 1$ pour permettre un développement maximum du compartiment de la cellule souche. Ce développement a été évalué chaque semaine en prélevant un aliquot que l'on repiquait en culture secondaire sur un milieu semi-solide afin de déterminer les différents progéniteurs produits. Après sept semaines de culture liquide, la production totale des différents types de progéniteurs (BFU-E, CFU-GM*) dans le sang de cordon s'est révélée significativement supérieure à celle de la moelle osseuse [12]. Ces résultats apportent une preuve supplémentaire que le compartiment de la cellule souche est constitué par des cellules hétérogènes ayant des potentiels hématopoiétiques plus ou moins larges. Sans doute faudra-til définir à l'avenir des souspopulations de cellules souches, tout comme on a défini des souspopulations de cellules $\mathrm{T}$ ou de macrophages $\left(\mathrm{m} / \mathrm{s} n^{\circ} 6-7\right.$, vol. 9, p. 772).

Aujourd'hui, l'objectif est de choisir des sources de cellules souches permettant de créer facilement des banques comportant un maximum de types HLA. De telles banques remplaceraient les registres de donneurs de moelle osseuse car ceuxci ne peuvent satisfaire qu'un faible nombre de demandeurs de greffe qui doivent être de plus "blancs caucasiens ", les autres ethnies n'étant pratiquement pas représentées dans ces registres. L'avantage du sang de cordon ombilical est qu'il est disponible sur toute la planète et plus facile à prélever que le sang périphérique ou la moelle osseuse. Des problèmes techniques restent à résoudre : (1) faudra-t-il congeler des poches de $150 \mathrm{ml}$ de sang de cordon ou pourra-t-on faire des banques de cellules CD34+ qui contiennent le compartiment des cellules souches et qui pourraient être congelées en tubes de 2 à $5 \mathrm{ml}$ ?; (2) faudra-t-il greffer les cellules souches purifiées avec cer-

- BFU-E : burst forming unit-erythroid; CFUGM : colony forming unil-granulo-monocyles. 
taines sous-populations cellulaires qui favorisent la destruction des cellules malignes (phénomène de graft versus leukemia ou GVL) sans provoquer le phénomène de rejet de greffe (graft versus host ou GVH) ? ; (3) pourra-t-on activer une partie seulement des cellules souches ou les associer à des progéniteurs plus mûrs pour accélérer la prise du greffon et ainsi réduire le coût de l'hospitalisation? Quel sera le rôle des cytokines dans ce cas? ; (4) la cellule souche est un candidat de choix pour la thérapie génique des maladies génétiques, des cancers et du sida. Pourra-t-on sortir de la phase Go les cellules souches purifiées, les activer pour pratiquer le transfert de gène, puis les remettre en phase quiescente pour les empêcher de se différencier?

Lorsqu'il y a trois ans, l'Action Concertée Européenne human bone marrow stem cell a commencé à étudier la possibilité de purifier la cellule souche pour l'utiliser à des fins cliniques, y compris en thérapie génique, le projet paraissait utopique pour beaucoup. Lorsqu'en novembre 1991, cette action concertée a invité des cliniciens européens à discuter de l'utilisation de cellules purifiées CD34+ pour la transplantation, la plupart d'entre eux sont restés prudents, attendant que d'autres démontrent l'intérêt de cette technique. Maintenant, ces projets sont devenus une réalité dans de nombreux centres de transplantation. Il est probable que, dans les prochaines années, la réalité dépassera largement les espoirs sous-jacents aux vieux mythes de la cellule souche

Jacques Hatzfeld, Béatrice Panterne, Patricia Sansilvestri, Pascal Batard, Jean-Pierre Lévesque, Angelo Cardoso, Ma-Lin Li, Michèle Ginsbourg, Yi-Qing Zhou, Antoinette Hatzfeld

Laboratoire de biologie cellulaire et moléculaire des facteurs de croissance, Cnrs, UPR 272, 7, rue Guy-Môquet, BP 8, 94801 Villejuif Cedex, France.

\section{TIRÉS A PART}

\section{J. Hatzf eld.}

\section{RÉFÉRENCES}

1. Baum CM, Weissman IL, Tsukamoto AS, Buckle AM, Peault B. Isolation of a candidate human hematopoietic stemcell population. Proc Natl Acad Sci USA 1992 ; 89 : 2804-8.

2. Till JE, McCulloch EA, Siminovitch L. A stochastic model of stem cell proliferation based on the growth of spleen colonyforming cells. Proc Natl Acad Sci USA 1964; 51 : 29-36.

3. Ogawa M. Differentiation and proliferation of hematopoietic stem cells. Blood 1993 (sous presse).

4. Panterne B, Zhou YQ, Hatzfeld J, Li ML, Lévesque JP, Clark SC, Hatzfeld A. CSF-1 control of c-fms expression in normal human bone marrow progenitors. J Cell Physiol 1993 ; 155 : 282-9.

5. Walker F, Nicola NA, Metcalf D, Burgess AW. Hierarchical down-modulation of hemopoietic growth factor receptors. Cell $1985 ; 43$ : 269-76

6. Metcalf D. Clonal analysis of proliferation and differentiation of paired daughter cells : action of granulocyte-macrophage colony-stimulating factor on granulocytemacrophage precursors. Proc Natl Acad Sci USA 1980; 77 : 5327-30.

7. Panterne B, Hatzfeld A, Zhou YQ, Li ML, Sansilvestri P, Cardoso A, Lévesque J-P, Ginsbourg M, Batard P, Kiselev $S$, Hatzfeld J. Contrôles positifs et négatifs du développement des progéniteurs hématopoïétiques : modèles et faits. Nouv Rev Fr Hematol 1993 ; 35 : 281-3.

8. Li ML, Cardoso A, Sansilvestri P, Hatzfeld A, Batard P, Lévesque JP, Hatzfeld J. Co-stimulatory effects of Steel Factor, the kit-ligand, on purified human hematopoietic progenitors in low cell density culture. Nouv Rev Fr Hematol 1993 ; 35 : 81-6.

9. Guigon $M$, Lemoine $F$, Dainiak $N$, Schechter A, Najman A. The negative regulation of hematopoiesis : from fondamental aspects to clinical applications. Paris : Inserm John Libbey Eurotext, 1993 (sous presse).

10. Hatzfeld J, Li ML, Brown EL, Sookdeo $\mathrm{H}$, Levesque JP, O'Toole T, Gurney C, Clark SC, Hatzfeld A. Release of early hematopoietic progenitors from quiescence by antisense transforming growth factor $\beta 1$ or $\mathrm{Rb}$ oligonucleotides. J Exp Med $1991 ; 174$ : 925-9.

11. Huang S, Terstappen LWMN. Formation of haematopoietic microenvironment and haematopoietic stem cells from single bone marrow stem cells. Nature 1992 ; 360 : 745-9.

12. Cardoso AA, Li ML, Batard P, Hatzfeld A, Brown EL, Levesque JP, Sookdeo H, Panterne B, Clark SC, Hatzfeld J. Release from quiescence of CD34+ CD38human umbilical cord blood reveals their potentiality to engraft adults. Proc Natl Acad Sci USA 1993 ; 90 : 8707-11. 